\title{
Intensifying chitin hydrolysis by adjunct treatments - an overview
}

Article

Accepted Version

Zainal Abidin, M., Junqueira-Gonçalves, M. P., Khutoryanskiy, V. and Niranjan, K. (2017) Intensifying chitin hydrolysis by adjunct treatments - an overview. Journal of Chemical Technology and Biotechnology, 92 (11). pp. 2787-2798. ISSN 0268-2575 doi: https://doi.org/10.1002/jctb.5208 Available at https://centaur.reading.ac.uk/68864/

It is advisable to refer to the publisher's version if you intend to cite from the work. See Guidance on citing.

To link to this article DOI: http://dx.doi.org/10.1002/jctb.5208

Publisher: Wiley

All outputs in CentAUR are protected by Intellectual Property Rights law, including copyright law. Copyright and IPR is retained by the creators or other copyright holders. Terms and conditions for use of this material are defined in the End User Agreement.

\section{www.reading.ac.uk/centaur}

\section{CentAUR}

Central Archive at the University of Reading 
Reading's research outputs online 


\section{Intensifying chitin hydrolysis by adjunct treatments - an overview}

Munira Zainal Abidin ${ }^{1,3}$, Maria Paula Junqueira-Gonçalves², Vitaliy V. Khutoryanskiy ${ }^{4}$, Keshavan Niranjan ${ }^{1 *}$

1Department of Food and Nutritional Sciences, University of Reading, Whiteknights, PO Box 226, Reading RG6 6AP, UK.

${ }^{2}$ Department of Food Science and Technology, University of Santiago, Chile. Ecuador 3769, Estación Central, Santiago, Chile.

${ }^{3}$ Department of Technology and Heritage, Universiti Tun Hussein Onn Malaysia, 86400, Parit Raja, Johor, Malaysia. ${ }^{4}$ Department of Pharmacy, University of Reading, Whiteknights, PO Box 224, Reading RG6 6AD, UK.

\section{Abstract}

Chitin is, after cellulose, the most abundant organic natural polysaccharide on Earth,

4 being synthesized as a dominant component in the exoskeletons of crustaceans, among other sources. In the processing of seafood for human consumption, between 40 and $50 \%$ of the total raw material mass is wasted, causing a significant problem 7 for the environment due to its slow degradation. Efforts to find uses for chitin 8 derivatives, particularly their oligomers, have intensified since these chemicals are 9 highly functional and offer a wide range of applications, especially as antimicrobial agent. As a consequence, some adjunct treatments, either chemical or physical in

\footnotetext{
${ }^{*}$ Corresponding author: Tel: +44 (0) 1183788388

E-mail: afsniran@reading.ac.uk (K. Niranjan)
} 
1 nature, have been employed to assist acid and enzymatic hydrolysis. This work

2 provides a detailed review of the methods employed to intensify the formation of

3 chitin oligomers, particularly focusing on the adjunct treatments used (microwave,

4 ultrasonication, steam explosion and gamma irradiation), and evaluate the yield and

5 characteristics of the oligomers formed. Adjunct treatments are more suitable for

6 enzymatic hydrolysis since these treatments modify the chitin structure, and enhance

7 the hydrolysis rate and yield of the oligomers, under milder reaction conditions. For

8 future research, it would be worth trying pre-treatments like the application of high-

9 pressure to chitin in order to lower its crystallinity.

10 Keywords: Chitin hydrolysis; Oligomers; acid hydrolysis; enzymatic hydrolysis;

11 pretreatment

\section{INTRODUCTION}

13 Chitin - a $\beta$-(1 $\rightarrow 4)$-linked polymer composed predominantly of $N$-acetyl-D14 glucosamine (GlcNAc) units - is, after cellulose, the most abundant organic natural polysaccharide on Earth, offering a broad range of structural and protection functions, like cellulose in plants. The structure of chitin differs from that of cellulose

17 in that the C-2 hydroxyl residues $(-\mathrm{OH})$ are replaced by acetamide groups $18\left(\mathrm{CH}_{3} \mathrm{CONH}-\right)$ (Figure 1). Chitin comprises three polymorphs: $\alpha$-chitin is the most 19 abundant and $\beta$ - and $\gamma$-chitin are very rare, which can be distinguished by their molecular chain arrangement and hydrogen bonding systems. $\alpha$ - and $\beta$-Chitins have

21 an antiparallel and a parallel chain arrangement, respectively, whereas $\mathrm{Y}$-chitin 22 consists of both parallel and antiparallel chains. ${ }^{1,2}$ The antiparallel chain in a-chitin 23 are arranged in bonded piles or sheets linked together by the hydrogen and 
1 acetamide groups running in opposite directions as compared to the $\beta$-chitin. ${ }^{2}$ The

2 intermolecular and hydrogen bonds present in a-chitin make it difficult to melt and

3 dissolve in common solvents at normal temperatures, which makes this material

4 inconvenient for further processing. ${ }^{1}$

5 Chitin is synthesized as a dominant component in the exoskeletons of crustaceans

6 and insects, as well as in the cell wall of fungi, yeast and algae. Nowadays, chitin

7 extracted from crustacean shells, such as crab, shrimp, prawn, krill and lobster, are

8 readily available in large quantities from shellfish processing industries in

9 comparison with other sources. In the processing of seafood for human

10 consumption, between 40 and $50 \%$ of the total raw material mass is wasted. ${ }^{3}$ This

11 quantity of waste has been reported to be between $10^{10}$ and $10^{11}$ tons per year,

12 which poses a significant problem for the environment due to its slow degradation. ${ }^{3-6}$

13 Crustacean shells are composed of proteins, chitin, minerals, and carotenoids, which

14 are the major components based on the dry mass. ${ }^{3}$ In order to extract chitin from the

15 crustacean shells, the following main steps must be employed: demineralization,

16 deproteinization and decolouration. ${ }^{7}$ The demineralization of shells can be achieved

17 by extraction with dilute acid (hydrochloric acid, formic acid, acetic acid, sulfuric acid

18 or EDTA - ethylenediamine-tetra-acetic acid) at room temperature. Deproteinization

19 can be effected by treating the demineralized waste with aqueous alkali solution

20 (sodium or potassium hydroxide) at the temperatures between 65 and $100^{\circ} \mathrm{C}$. In this

21 step, the most significant parameters to be considered for an efficient

22 deproteinization are the concentration of alkali solution, processing time and

23 temperature, and solid to solvent ratio. Benhabiles et al. ${ }^{8}$ reported that the conditions

24 for $96 \%$ protein removal were: processing time of 120 mins at a temperature of 45 
$1{ }^{\circ} \mathrm{C}$ and the use of solid to solvent ratio of $1: 2(\mathrm{w} / \mathrm{v})$ with the solvent $(\mathrm{NaOH}$ solution)

2 concentration being 2 M. Finally, the decolouration was carried out by a bleaching 3 with activated charcoal, or by using strong oxidizing agents such as sodium 4 hypochlorite $(\mathrm{NaClO})$ or hydrogen peroxide $\left(\mathrm{H}_{2} \mathrm{O}_{2}\right)$ solutions. ${ }^{9}$ Chitin separated from 5 minerals, colourants and proteins is commercially available for industrial uses, 6 although, it may require further purification in order to obtain regenerated chitin, 7 which can be effectively used for producing chitosan and oligomers. ${ }^{10-12}$

8 Chitosan - a $\beta$-(1 $\rightarrow 4)$-linked polymer composed predominantly of D-glucosamine 9 (GlcN) units - is an $N$-deacetylated derivative of chitin formed by deacetylation under 10 alkaline conditions at elevated temperature, which sodium hydroxide is a common 11 alkaline media. ${ }^{4,13-15}$ Chitosan is a semi-crystalline polymer which is insoluble in 12 aqueous solutions above a $\mathrm{pH}$ value of 6.5 , but fully soluble in diluted acids below $13 \mathrm{pH}$ of $5 .{ }^{16,17}$ The difference between chitin and chitosan can be defined in terms of 14 the ratio of 2-acetamido-2-deoxy-D-glucopyranose to 2-amino-2-deoxy-D15 glucopyranose units, which is commonly known as degree of $N$-acetylation (DA). The 16 DA of chitosan is typically less than 0.35 , whereas that of chitin is normally above $170.90 .^{15}$ Higher and lower molecular weight chitosans may also possess excellent properties for specific applications. Numerous studies have reported that chitosan with higher molecular weight possess superior mechanical properties, such as higher tensile strength and better elongation of chitosan film, ${ }^{18,19}$ higher antifungal 21 activity, ${ }^{20,21}$ and enhances nasal absorption of peptide drugs ${ }^{22}$. Lower molecular 22 weight chitosans have a stronger superoxide scavenging activity, ${ }^{23}$ greater antimicrobial activity, ${ }^{24}$ and higher permeability of film. ${ }^{18}$ 
1 Chitin oligomers are derived from chitin by depolymerization in the presence of acid

2 or enzymes. ${ }^{4,13,14}$ Chitin oligomers are composed of GlcNAc units with approximately

3 ten residues or less, and can be produced by depolymerization of chitin. ${ }^{4}$ The

4 depolymerization is commonly achieved by acid and enzymatic hydrolysis,

5 employing hydrochloric acid $(\mathrm{HCl})$ and chitinases, respectively. The chitin polymer

6 chain is cleaved by this reaction to become oligomeric. Both these hydrolysis

7 methods have been extensively reviewed by Jeon et al., Prashanth and Tharanathan

8 and Ahmed et al.4,25,26 Efforts to find uses for chitin oligomers have intensified since

9 these chemicals are highly functional and offer a wide range of applications. Chitin

10 oligomers have received increased research and commercial attention because

11 these molecules are not only water soluble, nontoxic and biocompatible, but also

12 exhibit numerous biological properties, such as antibacterial, antifungal, antitumor,

13 and antioxidant activities. ${ }^{27,28-30}$ According to the literature, antimicrobial action is one

14 of the most important property of chitin oligomers due to its water solubility, and

15 potent activity against bacteria and moulds. It has been reported that the oligomer

16 possess low minimal inhibitory concentrations (MIC) values for gram-positive strain

17 (S.aureus, B. subtilis, L. monocytogenes and B. cereus) and gram-negative strains

18 (E. coli, V. cholera, Shigella disenteriae, B. fragilis, $P$. aeruginosa, and $P$.

19 melaninogenica) compared to chitin and chitosan. ${ }^{27,31}$ In another research, the chitin

20 oligomers were found to possess antifungal activity against Aspergillus niger. ${ }^{28}$

21 Besides antimicrobial activity, some works also reported that the chitin oligomers,

22 with molecular weight of 1-5 kDa, possess the ability to inhibit membrane protein

23 oxidation and act as potent antioxidant in live cells. ${ }^{30,32}$ 
1 As a consequence of growing interest for converting chitin into its oligomers, some

2 adjunct treatments, either chemical or physical in nature, have been employed to

3 assist acid and enzymatic hydrolysis, as well as enhance the yield of oligomers.

4 Gamma irradiation, ultrasonication, microwave irradiation, steam explosion,

5 supercritical water, grinding and depressurization are physical methods that have

6 been employed previously to facilitate chitin hydrolysis. ${ }^{33-38}$ Aqueous solutions such

7 as phosphoric acid, hydrochloric acid, alkaline solution and methanol modify chitin

8 structures prior to hydrolysis. These chemical treatments have been reported to

9 decrystallise chitin, increase its solubility, and accelerate subsequent enzyme 10 hydrolysis. ${ }^{11,12,39}$

11 This article aims to review the methods which have been employed to intensify the

12 formation of chitin oligomers, particularly focusing on the adjunct treatments to

13 improve the hydrolysis, and evaluate the characteristics of the chitin and oligomers 14 formed.

\section{PREPARATION OF CHITIN OLIGOMERS}

16 This section reviews the different hydrolysis methods employed and adjunct 17 treatments proposed to assist depolymerization of chitin.

18 Methods based on acidic hydrolysis

19 Chitin oligomers are generally manufactured by acid hydrolysis of chitin employing 20 strong acids such as concentrated hydrochloric acid $(\mathrm{HCl})$ to effect the cleavage of 21 the chitin polymeric chain. ${ }^{27,30,34,40-42}$ The concentration of $\mathrm{HCl}$, incubation time and 22 temperature are the key parameters in the process. $\mathrm{HCl}$ concentrations ranging from 233 to $12 \mathrm{~N}$ have been used to hydrolyze chitin at temperatures ranging between 20 
1 and $90 \stackrel{\circ}{\circ}$ for time durations ranging between 5 min and 7 h. ${ }^{27,30,32,40-42,44,45}$ The

2 processing steps employed by earlier researchers for hydrolysing chitin are

3 summarized in Figure 2. In general, a given amount of chitin powder is added to $\mathrm{HCl}$

4 solution and the mixture is constantly stirred under reflux in a water bath maintained

5 at the desired temperature. ${ }^{32,40,42}$ After incubating thus for the desired time period,

6 the hydrolysis is stopped by cooling the reaction mixture in an ice bath or on dry

7 ice,,$^{40,44}$ following which, the chitin oligomers are isolated by: 1) freeze-drying the

8 solution under vacuum, 2) redissolving the dried product in deionised water, and 3)

9 neutralising the solution with $\mathrm{NaOH}$ followed by filtration to remove impurities from

10 the oligomers. The freeze-drying and redissolving steps have been repeated twice

11 by some researchers to remove any residual $\mathrm{HCl}$ left. ${ }^{42}$ Some researchers have also

12 proposed neutralisation with $25 \%$ sodium hydroxide $(\mathrm{NaOH})$ to stop the

13 hydrolysis. ${ }^{30,32}$ The neutralized solution is centrifuged to separate the supernatant,

14 and the unhydrolyzed chitin and the supernatant are desalted and purified prior to

15 spray-drying in order to obtain the chitin oligomers as a light yellow powder.

16 Recently, Kazami et al. ${ }^{45}$ developed an acetone precipitation method as a

17 replacement to $\mathrm{NaOH}$ neutralisation to claim a simpler procedure for isolating the

18 chitin oligomers. Acetone-insoluble material can be recovered by the following steps:

19 1) adding acetone to stop the hydrolysis and stirring at a low temperature for a day;

20 2) centrifuging the mixture of acetone and chitin; 3) repeatedly washing the acetone-

21 precipitate with acetone to remove $\mathrm{HCl}$ (until the $\mathrm{pH}$ of supernatant reaches 4-5);

22 and 4) suspending the acetone-precipitate in cold diethyl ether, centrifuging and 23 drying to constant weight. 
1 The dried acetone-precipitate (acetone-insoluble material) is then mixed with water

2 in order to extract the water-soluble chitin oligomers as follows: 1) mixing the dried

3 acetone-precipitate with water, stirring overnight, and centrifuging in order to

4 separate the supernatant and water-precipitate; 2) repeating step (1) for the water-

5 precipitate; 3 ) combining supernatant recovered from steps (1) and (2) as water-

6 soluble chitin oligomers; and 4) suspending the water-precipitate in cold diethyl

7 ether, centrifuging and drying to constant weight. Generally, the supernatant

8 containing the chitin oligomers obtained appear brown or yellow in colour. Therefore,

9 activated charcoal treatment is applied to yield a clear solution prior to the final

10 drying process. ${ }^{34,42}$

11 Chitosan can also be potentially converted into chitin oligomers by employing a two-

12 step process involving: 1) depolymerization of chitosan by hydrolysis in $\mathrm{HCl}$ to form

13 chitosan oligomers; and 2) partial $\mathrm{N}$-acetylation of chitosan oligomer in hydro-

14 alcoholic solution of acetic anhydride in order to produce the oligomers. ${ }^{42}$ This

15 approach generally requires numerous steps and produces $\mathrm{HCl}$ and acetic anhydride

16 residues, which are undesirable products and have a significant impact on the

17 environment.

18 Figure 3 shows a possible mechanism of chitin hydrolysis by concentrated $\mathrm{HCl}$ that 19 has been reported previously by Kazami et al. ${ }^{45}$ The chitin before hydrolysis is 20 assumed to consist of alternating crystalline and amorphous regions, and composed 21 a number of polymeric chains. Initially, the amorphous regions are rapidly cleaved 22 within $5 \mathrm{~min}$ of hydrolysis to produce regular-sized segments with a central 23 crystalline region attached to amorphous tails at both ends. The amorphous tails are 24 then gradually degraded, leading to the accumulation of chitin oligomers, as well as 
1 a crystalline chitin core consisting of multiple chitin chains. Single chitin chains may

2 then be slowly separated from the chitin core, and once separated, be rapidly

3 hydrolyzed to yield chitin oligomers within 30 to $60 \mathrm{~min}$.

4 Although the hydrolysis process described above is effective, some disadvantages

5 have been reported such as the occurrence of deacetylation (that produces chitosan

6 oligomers instead of chitin oligomers), production of acidic waste streams, high cost,

7 lower yield of high degree of polymerization (DP) oligomers, and requiring skilled

8 labor force for purification. $4,11,25$ The lower yield and shorter chain length of the

9 oligomers formed, particularly dimers, adversely influence bioactivity. ${ }^{46}$ In addition,

10 the process costs and the environmental impact of the process are also high mainly

11 due to the use of strong acids during hydrolysis. ${ }^{6}$

\section{Methods based on enzymatic hydrolysis}

13 In contrast to acidic hydrolysis, higher DP chitin oligomers can be produced under

14 milder reaction conditions by employing enzymatic hydrolysis. Enzymes, mainly 15 chitinases, which have higher chitinolytic activity, are used for this purpose, and are 16 commonly produced from microorganisms, plants, and insects. Some researchers 17 have used bacterium Serratia proteamaculans $568,{ }^{47}$ Serratia marcescens $2170,{ }^{48}$ 18 Rhizobium sp. GRH2,49 Bacillus cereus $\mathrm{TKU}^{49} 2,{ }^{6}$ to produce chitinases, while 19 others have used hevamine, ${ }^{50}$ a plant enzyme, having both chitinase and lysozyme 20 activities. Chitinases can also be successfully extracted from fungi such as

21 Lecanicillium lecanii and Lecanicillium fungicola, while Trichoderma reesei fungi is 22 reported to have hydrolases (cellulases and $\beta$-glucanases). ${ }^{11,36,39}$ The enzymes 23 produced from various sources have to be purified before being used for hydrolysis. 
1 Additionally, non-chitinase commercially available enzymes, like cellulase,

2 hemicellulase, pepsin, papain, lysozyme, and pectinase have also been reported to 3 hydrolyse chitin. ${ }^{12,31,51}$

4 A flow diagram for the enzymatic hydrolysis of chitin is showed in Figure 4. Prior to 5 hydrolysis, the substrate or chitin suspension is prepared by adding chitin powder to 6 a phosphate or acetate buffer solution, so that its concentration is between 0.5 and $72.0 \% \mathrm{w} / \mathrm{v} \cdot{ }^{6,12,33,48,51}$ Buffers strength in the range of 0.01 to $0.05 \mathrm{M}$ and $\mathrm{pH} 5.0$ to 5.5 , 8 are reported to provide optimum condition for the substrate preparation. $6,12,31,36,51$

9 The enzyme is subsequently mixed with the substrate at an appropriate amount, so 10 that its chitinolytic activity in the system is under the standard assay condition. In the 11 hydrolysis, one unit of enzyme activity $(1 \mathrm{U})$ is defined as the amount of enzyme 12 releasing $1 \mu \mathrm{mol}$ GlcNAc per minute. ${ }^{52}$ After mixing, the mixture is incubated at various temperature-time combinations, depending on the enzyme action. Hydrolysis by chitinases, lysozyme, pectinase, and pepsin have been reported to require incubation at temperatures between 37 and $44{ }^{\circ} \mathrm{C} .6,12,36,47,48,50,51$ To stop the reaction, the hydrolysis mixture is heated to $90^{\circ} \mathrm{C}$ or boiled for $10 \mathrm{~min}$, and subsequently

17 centrifuged and filtered to separate the supernatant which contains the oligomers, and unhydrolysed chitin. ${ }^{11,31,36,51}$

19 Earlier studies have reported that the yield of high DP chitin oligomer resulting from enzymatic hydrolysis is greater compared to acidic hydrolysis. ${ }^{4}$ This may be due to 21 the enzyme acting selectively on the crystalline and otherwise inaccessible parts of 22 chitin. During chitin hydrolysis, the enzymes which degrade the polysaccharide chain 23 can be either endo-acting or exo-acting. Figure 5 shows the mechanism of endoacting enzymes which randomly cleave glycosidic linkages of chitin, generate free 
1 ends and chitin oligomers, while exo-acting enzymes release dimers (two units of

2 GlcNAc) from the reducing (C1) or non-reducing (C4) ends. ${ }^{47}$

3 Of course, the use of enzymes also has its fair share of disadvantages: specific

4 enzymes such as chitinase and chitosanases are not readily available commercially 5 and, even if available, tend to be very expensive. ${ }^{37}$ Further, the presence of protein 6 residues after hydrolysis potentially limit biomedical application due to possible 7 allergen and pyrogenicity ${ }^{25}$ effects, which will warrant significant further purification

8 that will make the whole process economically unviable. However, this method has a 9 key advantage because minimum chemical wastes are produced during hydrolysis. ${ }^{6}$

\section{Use of chemical and physical adjunct treatments to intensify chitin hydrolysis}

11 Irradiation of chitin has recently been used to assist acid or enzymatic hydrolysis.

12 Ultrasonic irradiation or ultrasonication has been reported to be advantageous for 13 depolymerization, because it preserves the chemical nature of the polysaccharide by 14 simply splitting the most susceptible chemical bonds and lowering its molecular weight. ${ }^{53}$ Takahashi et al..$^{54}$ and Ajavakom et al. ${ }^{34}$ determined the effectiveness of ultrasonication during acid hydrolysis. In these investigations, the mixture of chitin and $\mathrm{HCl}$ was sonicated at various wavelengths for different durations. It has been

18 found that the chitin powder completely dissolved within 30 min in the $\mathrm{HCl}$ solution 19 during sonication at 50 or $60 \mathrm{~Hz}(275 \mathrm{~W}) .{ }^{34}$ Takahashi et al. ${ }^{54}$ noted that the amount of oligomers up to DP 7 (seven units of GlcNAc in each chain) increased after 120 min of ultrasonication, which was 2 to 4 times greater than oligomers produced

22 without ultrasonication. On the other hand, the chitin could be degraded during 23 demineralization in an ultrasound-assisted extraction process. The depolymerization 
1 occurring may be due to the application of high intensity irradiation, which results in

2 breaking covalent bonds in the polymeric chain. ${ }^{55}$ Ultrasonication has also been

3 applied to the chitin suspension prior to enzymatic hydrolysis. In this process, the

4 treated chitin depolymerized to a lesser extent, which was detected by the lower

5 amount of reducing sugars measured. ${ }^{36}$ All these studies agreed that ultrasonication

6 facilitated acidic and enzymatic hydrolysis without drastically changing the degree of

7 acetylation (DA) of the chitin. However, if high intensity ultrasonication was applied,

8 the covalent bond in the polymeric chains of chitin could break due to cavitation by

9 temporarily dispersing aggregates. ${ }^{55}$

10 Some researchers have measured ultrasonic intensity directly, while others have not.

11 When the intensity is not measured, it is calculated by measuring the transient rise in

12 temperatures during ultrasonication, and after it is switched off, as follows:

$$
I=\frac{m c_{p}}{\pi r^{2}}\left[\left(\frac{\mathrm{d} T}{\mathrm{~d} t}\right)_{a}-\left(\frac{\mathrm{d} T}{\mathrm{~d} t}\right)_{b}\right]
$$

14 where: $I$ is the ultrasonic intensity, $(d T / d t)_{a}$ is the slope of the initial rise in 15 temperature, $(\mathrm{d} T / \mathrm{d} t)_{\mathrm{b}}$ is the slope of heat loss after the ultrasonic processor was 16 turned off, $m$ is the sample mass, $c_{p}$ is the heat capacity of the solvent, and $r$ is the 17 radius of ultrasonic probe. ${ }^{55}$

18 Microwave irradiation has been established as a patented technique for producing chitin oligomers. Chitin is added to $\mathrm{HCl}$ and subjected to a conventional microwave device at 700 to $2100 \mathrm{~W}$ for up to $24 \mathrm{~h} .{ }^{56}$ This technique has been repeated by other 21 researchers with a slight modification, where $38 \% \mathrm{HCl}$ was initially pre-warmed at $22850 \mathrm{~W}$ by conventional microwave oven for a shorter time and the pre-warmed $\mathrm{HCl}$ 
1 was quickly added to chitin powder for further irradiation at various reaction times. ${ }^{34}$

2 Microwave irradiation has also been applied prior to the enzymatic hydrolysis of

3 chitin. Roy et $a{ }^{33}$ used a microwave with built-in magnetic stirrer and non-contact

4 infrared continuous feedback temperature system for chitin pre-treatment. In this

5 study, the chitin suspended in acetate or phosphate buffer was irradiated with the

6 microwave at optimum temperature and time reported to be $57.5 \stackrel{\circ}{\circ}$ and $38 \mathrm{~min}$,

7 respectively; this was followed by hydrolysis with chitinase. This study found that the

8 polar molecules in the chitin suspension align with the magnetic field generated by

9 microwave, and have a tendency to accelerate the hydrolytic reaction rate. The

10 microwave pre-treatment is comparable to ultrasonication, when the treated chitin is

11 insignificantly deacetylated after irradiation. ${ }^{33}$

12 Improvement on the hydrolysis rate and chitin properties could be accomplished by

13 gamma irradiation, which is one of the physical methods requiring no chemical

14 additive and no temperature control during reaction. ${ }^{37,38}$ Gamma irradiation, applied

15 at different doses ranging from 15 to $210 \mathrm{kGy}$, to the solid form of chitin, prior to

16 hydrolysis with chitinases, has been investigated. ${ }^{37}$ Previously, this method was

17 effectively applied as the adjunct treatment in the production of chitosan oligomer,

18 using irradiation doses ranging between 2 and 500 kGy. ${ }^{57-59}$ These authors

19 suggested that the application of gamma irradiation at various doses may reduce the

20 molecular weight of oligomers due to the breaking of glycoside bond.

21 The adjunct treatments discussed above are physical methods which influence the

22 reaction through non-thermal effects. However, thermal treatments, such as steam

23 explosion (SE), has also been investigated to influence the enzymatic hydrolysis of

24 chitin. Steam explosion treatment consists in heating of chitin with saturated steam, 
1 followed by a sudden decompression of the pressurized system to produce insoluble

2 solid fraction and a liquid fraction of soluble sugars. ${ }^{60,61}$ Villa-Lerma et al. ${ }^{36}$ applied

3 steam explosion to the mixture of chitin powder and deionized water at $180 \stackrel{\circ}{\circ} \mathrm{C}$ and 1

$4 \mathrm{MPa}$ for various reaction times. The treated chitin mixture was then added to

5 phosphate buffer and this combination acted as substrate for hydrolysis with

6 chitinase. It has been reported that steam explosion can significantly reduce chitin

7 crystallinity without significant depolymerization occurring during treatment.

8 Another physical treatment that has been proposed to enhance and increase the

9 oligomers yield is a combination of the use of supercritical water and mechano10 chemical grinding with a ball mill. ${ }^{35}$ Supercritical water is water at temperatures near 11 or above $374{ }^{\circ} \mathrm{C}$, meanwhile, the mechano-chemical grinding is a term used for the 12 chemical reaction that occurs during mechanical treatment of the sample, typically 13 grinding by ball mill.62-64 After being treated with supercritical water, the chitin 14 undergoes mechano-chemical grinding. This pre-treatment results in chitin flakes 15 which are fragile and easy to grind, and form an effective substrate for hydrolysis 16 with reduced particle size and molecular weight. ${ }^{35}$

17 In addition, chitin structures can also be pre-treated chemically by using aqueous 18 solution, such as phosphoric acid, $\mathrm{HCl}$, alkaline solution (mixture of sodium 19 hydroxide and sodium dodecylsulfate), and methanol. These pre-treatments have reportedly decrystallized chitin, increased its solubility, and accelerated subsequent 21 enzyme hydrolysis. ${ }^{11,12,39}$ Ramírez-Coutiño et al. ${ }^{39}$ deacetylated $\alpha$ and $\beta$-chitin by 22 deacetylation with alkali solution, thereby partially transforming chitin into chitosan 23 (degree of deacetylation 55 and $50 \%$, respectively) prior to hydrolysis. Reduction in 
1 hydrogen bonds caused by the elimination of acetyl group increases the solubility of

2 partially deacetylated chitin in aqueous media. ${ }^{65,66}$

\section{CHARACTERISTICS AND CHARACTERIZATION OF CHITIN AND ITS 4 OLIGOMERS}

5 This section summarizes the various methods used in literature to characterize chitin

6 oligomers produced by chitin hydrolysis with and without the use of adjunct 7 processes. Chemical structure and composition, degree of $\mathrm{N}$-acetylation (DA), 8 degree of polymerization (DP), molecular weight, and crystallinity are important 9 properties characterizing chitin oligomers. The methods employed to determine 10 these characteristics are discussed below.

\section{Chemical structure and composition}

12 Fourier transform infrared (FT-IR) and proton nuclear magnetic resonance H NMR 13 spectroscopies can be reliably used to record the composition and chemical 14 structure of the oligomers. ${ }^{30,32,34,36,37,39,41-43,55}$ These methods require simpler 15 procedures to prepare samples, and provide information on the chemical structure 16 faster than conventional methods. ${ }^{67}$

\section{Fourier transform infrared spectroscopy (FT-IR)}

18 FT-IR spectroscopy is a very attractive technique suitable for both soluble and 19 insoluble samples. ${ }^{68}$ The technique is based on the vibrations of the atoms of a 20 sample molecule. Infrared spectrum is obtained by passing infrared radiation through 21 the sample and determining the fraction of the incident radiation absorbed at a 
1 particular energy. The energy at any peak in an absorption spectrum corresponds to

2 the frequency of a vibration of a part of the sample. ${ }^{69}$

3 The infrared spectrum can be divided into three main frequencies which is far-

4 infrared $\left(<400 \mathrm{~cm}^{-1}\right)$, mid-infrared $\left(4000-400 \mathrm{~cm}^{-1}\right)$ and near-infrared $(13000-4000$

$5 \mathrm{~cm}^{-1}$ ). To obtain the FT-IR spectrum of the chitin and its oligomers, mid-infrared

6 frequency is used as explained by Lambert-Beer Law. $27,30,36,55,70$ The law states that

7 the absorbance values at a given frequency of 4000 to $400 \mathrm{~cm}^{-1}$ are proportional to

8 the concentrations of the sample. Measurements are initiated by preparing $\mathrm{KBr}$

9 (potassium bromide) pellet, which is a small amount of chitin sample well mixed with

10 the $\mathrm{KBr}$ (approximately 0.1 to $1.0 \% \mathrm{w} / \mathrm{w}$ ) and pressed into a pellet-forming die. The

11 measurement is taken by scattering the infrared light onto the $\mathrm{KBr}$ pellet using FT-IR

12 system. ${ }^{30,70,71}$ This is the most common method used, which utilizes the property of

$13 \mathrm{KBr}$ as alkali halide that becomes plastic when subjected to pressure and forms a

14 transparent sheet in the infrared region. ${ }^{72}$

15 In a chitin molecule, the acetamido group at the position C2 (Figure 5), 16 intramolecular hydrogen bonds $\left(\mathrm{C} 6-\mathrm{OH}^{\cdots} \mathrm{O}=\mathrm{C}\right)$ and intermolecular hydrogen bonds $17 \quad\left(\mathrm{NH}^{\cdots} \mathrm{C}=\mathrm{O}\right)$ provide polymer stability. ${ }^{73}$ In a form, the absorption bands of carbonyl 18 group (amide I) stretching are split at $1660 \mathrm{~cm}^{-1}$ and $1620 \mathrm{~cm}^{-1}$, which are attributed 19 to intermolecular and intramolecular hydrogen bonds respectively. ${ }^{73,74}$ Unlike a 20 crystalline form, a single absorption band of amide I $\beta$-chitin is observed at $1560 \mathrm{~cm}^{-}$ $21{ }^{1}$ which is attributed to the stretching of carbonyl group hydrogen bonded to amide 22 group of the intra-sheet chain. ${ }^{74}$ In previous research, a-chitin extracted from 23 Daphnia magna resting egg (zooplankton genus in aquatic ecosystems) exhibited 24 absorption bands at $1652 \mathrm{~cm}^{-1}$ and $1622 \mathrm{~cm}^{-1}$ for amide.$^{73}$ Recently, the chitin 
1 extracted from six different aquatic invertebrates presented the spectrum of amide I

2 at $1652-1656 \mathrm{~cm}^{-1}$ and $1619-1620 \mathrm{~cm}^{-1}$, thus allowing them to be characterized as a

3 crystalline form. ${ }^{75}$ These two strong absorption bands were also observed in

4 ultrasonication and steam explosion treated chitin. However, an additional shoulder

5 was present at $1633 \mathrm{~cm}^{-1}$ in ultrasonication treated chitin which might be due to a

6 reduction in amino-based hydrogen bonding. ${ }^{36}$ In chitin oligomers structure, the

7 spectrum generated by FT-IR exhibits intense absorption bands at $3358 \mathrm{~cm}^{-1}(\mathrm{OH}$

8 stretch), $2917 \mathrm{~cm}^{-1}$ (C-H stretch), $1651 \mathrm{~cm}^{-1}$ (C=O stretch, amide I), $1548 \mathrm{~cm}^{-1}(\mathrm{~N}-\mathrm{H}$

9 bend, amide II), $1313 \mathrm{~cm}^{-1}$ (C-N stretch, amide III), and 1150-1000 $\mathrm{cm}^{-1}$ (pyranose),

10 as shown in Figure $6 .{ }^{30}$ All the reported FT-IR spectra indicate that the crystalline

11 structure formation of intact chitin, treated chitin and its oligomers are not affected by

12 the types of processes.

\section{Proton nuclear magnetic resonance ( ${ }^{1} \mathrm{H}$ NMR) spectroscopy}

$14{ }^{1} \mathrm{H}$ NMR spectroscopy is a powerful and reliable technique for polymer structural 15 analysis by exploiting the magnetic properties of certain atomic nuclei. The spectra 16 of chitin and its oligomers can be obtained by cross-polarization or magic-angle17 spinning method using deuterated oxide $\left(\mathrm{D}_{2} \mathrm{O}\right)$ as solvent. ${ }^{31}$

18 In chitin, the chemical composition obtained by ${ }^{1} \mathrm{H}$ NMR resonated between 3.6 and 194.4 ppm, which are assigned to $\mathrm{H}-2$ to $\mathrm{H}-6$ protons, while acetyl protons are found at 202.6 ppm. $^{74,43}$ The anomeric region of $\alpha-$ and $\beta$-anomer $(\mathrm{H}-1)$ generated peaks at 5.43 21 and 5.05 ppm, respectively. ${ }^{43}$ As observed in chitin oligomers, the spectra present 22 two singlets at 2.06/2.08 ppm assigned to the $\mathrm{N}$-acetyl protons, and a broad signal at 235.20 ppm assigned to $\mathrm{H}-1$ protons of the reducing end $\alpha$ anomer residues. ${ }^{42}$ The 
1 chitin oligomers produced by enzymatic hydrolysis of chitin using commercial

2 enzymes possessed obvious resonance peak at $1.9 \mathrm{ppm}$ assigned to the acetyl

3 group, 3.4 ppm detected for $\mathrm{H}-2$ and 3.5 to 3.8 ppm corresponding to $\mathrm{H}-3, \mathrm{H}-4, \mathrm{H}-5$

4 and $\mathrm{H}-6$ protons, meanwhile the $\mathrm{H}-1(\beta)$ and $\mathrm{H}-1(\alpha)$ protons generated peaks at

5 around 4.6 to $4.7 \mathrm{ppm}$ and $5.2 \mathrm{ppm}$, respectively. ${ }^{31} \mathrm{Ngo}$ et al. ${ }^{30}$ reported the

6 resonance of chitin oligomers hydrolyzed by acid $(\mathrm{HCl})$ at $1.97 \mathrm{ppm}$ is assigned to

7 acetyl group, $3.34 \mathrm{ppm}$ is corresponded to $\mathrm{H}-2$, 3.37 to $3.77 \mathrm{ppm}$ are detected for $\mathrm{H}$ -

$83,4,5,6,4.5$ to 4.6 ppm is assigned to $\mathrm{H}-1(\beta)$ and 5.0 ppm is corresponded to $\mathrm{H}-1$

9 (a). All spectra of chitin are essentially identical to the oligomers, regardless of the

10 type of hydrolysis. It is caused by the occurrence of glyosidic linkages breakdown

11 during the hydrolysis without the interference of deacetylation. ${ }^{41}$

\section{Degree of $\mathrm{N}$-acetylation}

13 The degree of $\mathrm{N}$-acetylation (DA) represents the molar fraction of $\mathrm{N}$-acetylated units 14 in the chitin polymer chain. ${ }^{76}$ The DA is an important parameter influencing physico15 chemical, electrostatic and biological properties of chitin. ${ }^{77}$ Generally, the DA can be 16 determined by the calculation of the absorbance and intensities generated by FT-IR 17 and NMR, respectively. The absorbance values (A) obtained by FT-IR can be used 18 to measure DA by using the following equation:

$$
\% N \text {-acetylation }=\left(\mathrm{A}_{1655} / \mathrm{A}_{3450}\right)(100 / 1.33)
$$

where, $A_{1655}$ and $A_{3450}$ are the absorbances at $1655 \mathrm{~cm}^{-1}$ of the amide-I band and $213450 \mathrm{~cm}^{-1}$ of the hydroxyl band, respectively; and the factor of 1.33 is the ratio of

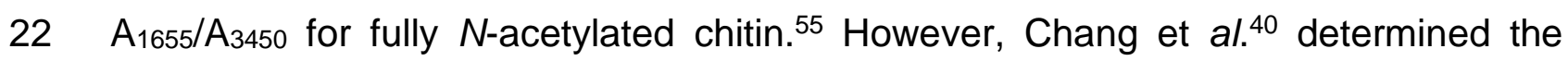


1 percentage of DA by using the following equation, originally proposed by Baxter et 2 al. ${ }^{78:}$

4 The values of $100 / 1.33$ (i.e. 75.2 ) and 115 are reciprocal values of the slope of the 5 linear section of the plot of absorption ratio $\left(A_{1655} / A_{3450}\right)$ against $D A$, which depend 6 on the baselines used. ${ }^{67,70} \mathrm{Eq}(3)$ is more reliable in comparison with Eq (2), since $7 \mathrm{Eq}(2)$ tended to overestimate values for $\mathrm{DA}>20 .{ }^{67}$

8 The DA can also be quantitatively analysed by carbon nuclear resonance magnetic 9 (C-NMR), where the relative intensities determined for the resonance of the ring 10 carbon $\left(I_{\mathrm{c} 1}, I_{\mathrm{c} 2}, I_{\mathrm{C}}, I_{\mathrm{C}}, I_{\mathrm{C}}, I_{\mathrm{C}}\right)$ and methyl carbon $\left(I_{\mathrm{CH}}\right)$ is used in the following 11 equation proposed by Ottey et al. ${ }^{79}$ :

$$
\% \mathrm{~N} \text {-acetylation }=\frac{I_{\mathrm{CH} 3}}{\left(I_{\mathrm{C} 1}+I_{\mathrm{C} 2}+I_{\mathrm{C} 3}+I_{\mathrm{C} 4}+I_{\mathrm{C} 5}+I_{\mathrm{C} 6}\right) / 6} \times 100
$$

13 Eqn (4) has been applied in earlier work on chitin. ${ }^{68,70,80}$

14 Fully acetylated chitin provides DA of $100 \%$ while $0 \%$ corresponds to completely 15 deacetylated chitin (chitosan). ${ }^{67}$ In previous work, the DA of chitin decreased from $1698 \%$ to 93,88 and $73 \%$ after exposure to ultrasonication, steam explosion and 17 depressurization, respectively. ${ }^{36,38}$ The lower DA values after adjunct treatment 18 indicate deacetylation and modification of chitin structure. However, the lowest DA of 19 depressurized chitin gave higher yield of the oligomers. It has been reported that the 20 DA values of chitin between 45 and $55 \%$ provide excellent solubility in aqueous 21 media. $^{38}$ Some authors have reported that the DA between 40 and $60 \%$ possessed 
1 suitable characteristics for enzymatic hydrolysis, where the chitin solubility reached

2 was greater than $60 \%{ }^{39,81}$ It caused the polarity and electrostatic repulsion of the

3 amino groups increased, thus increasing the accessibility of chitin to enzymatic

4 attack. ${ }^{39}$

\section{Degree of polymerization}

6 Degree of polymerization (DP) is a significant parameter to identify the number of

7 monomeric units in the oligomers. MALDI-TOF mass spectrometry is a powerful

8 technique, which has been extensively applied to determine the DP of chitin and

9 chitosan oligomers. ${ }^{6,36,42,82,83}$ Figure 7 shows the MALDI-TOF spectra which consists

10 of high intensity of a number of peaks assigned to a certain DP. The DP of the

11 oligomers can be determined by the peak-to-peak mass difference of 203, which is

12 the GlcNAc repeating unit $\left(\mathrm{C}_{8} \mathrm{H}_{13} \mathrm{NO}_{5}\right)$. The end-groups of $\mathrm{H}$ and $\mathrm{OH}$ are deduced

13 from monoisotopic mass. Each DP can be calculated from a peak with particular 14 mass unit $(\mathrm{m} / \mathrm{z})$ as follows:

$$
\mathrm{DP}=(\text { mass unit of a peak }-\mathrm{H}-\mathrm{OH}-\mathrm{Na}) / \mathrm{C}_{8} \mathrm{H}_{13} \mathrm{NO}_{5}
$$

where DP is the degree of polymerization. The molecular mass of $\mathrm{C}_{8} \mathrm{H}_{13} \mathrm{NO}_{5}$ is 203

17 (i.e. mass unit of chitin oligomers); the atomic mass of $\mathrm{H}$ is 1 (hydrogen); and those 18 of the $\mathrm{OH}$ group and $\mathrm{Na}$ are 17 and 22.99 , respectively. ${ }^{42}$

19 The DP can also be determined by using high performance liquid chromatography 20 (HPLC), where the concentration of each DP present can be calculated from the 21 peak areas in the HPLC profile using the standard curve obtained from pure chitin 22 monomer, dimer, trimer, tetramer, pentamer, and hexamer standard 23 solution. 6,11,12,40,57 Moreover, the DP can be quantitatively analysed by integrating the 
1 signals of the anomeric protons $(\mathrm{H}-1(\alpha)$ and $\mathrm{H}-1(\beta))$ obtained by ${ }^{1} \mathrm{H}$ NMR using the

2 following equation:

4 where $\int \mathrm{H}-1 \quad(\alpha)$ and $\int \mathrm{H}-1 \quad(\beta)$ refer to the integral of the $\mathrm{H} 1$ protons of the chitin 5 oligomers at terminal reducing end having $\alpha$ and $\beta$ configuration, respectively, and $6 \int \mathrm{H}-1 \mathrm{c}$ is the sum of integrals of the $\mathrm{H} 1$ protons of all the central units. ${ }^{84}$

7 As reported by Chang et al. ${ }^{40}$, the chitin hydrolysed in acid $(\mathrm{HCl})$ produced oligomers 8 with DP in the range of 2 to 6 . Moreover, the oligomers with DP 4-6 were simply 9 isolated from acetone-precipitation method. ${ }^{45}$ In another study, the chitin oligomers produced by acetylation of chitosan oligomers with various DA up to $90 \%$ using 11 acetic anhydride solution and isolated in $\mathrm{HCl}$ resulted in a value of $\mathrm{DP}$ ranging from 3 12 to 7.42 As an alternative to acid hydrolysis, enzymatic hydrolysis has been 13 extensively used to obtain oligomers with higher DP. As observed by Wang et al. ${ }^{6}$ 14 and Purushotham et $a l .{ }^{47}$, the oligomers obtained by hydrolysis with chitinases possessed DP in the range between 2 and 9 . In theory, the hydrolysis must ultimately lead to the formation of chitobiose (DP 2). However, products with DP 4 17 and higher show better functional properties as compared to DP 2. Chitinase and 18 lysozyme from hevamine were reported to degrade chitin polymer to form pentamers 19 (DP 5). ${ }^{70}$ Commercial enzymes such as hemicellulase and pectinase have also successfully depolymerized chitin to DP $6 .{ }^{31}$ Based on the above studies, it can be concluded that both acid and enzymatic hydrolysis result in higher DP of chitin 22 oligomers. However, the enzymatic hydrolysis condition is milder than acid 23 hydrolysis. 


\section{Crystallinity}

2 Chitin has a highly ordered crystalline structure, and degrading into oligomers may 3 reduce its crystallinity. Generally, the crystallinity of chitin and its oligomer can be

4 evaluated using $\mathrm{X}$-ray diffraction measurements. The peak intensity of chitin is 5 recorded over the scattering range of $4.5^{\circ}$ to $50^{\circ}$ with scan steps of $0.02^{\circ}$ at a speed 6 of $4.0^{\circ} \mathrm{min}^{-1} .^{36,45}$ The crystallinity index $\left(I_{\mathrm{CR}}\right)$ is generally measured by a method, 7 which uses a maximum intensity of $110\left(l_{110}\right)$ and the intensity of amorphous halo 8 contribution $(\mathrm{lam}),{ }^{36,45,55,85}$ as follows:

$$
I_{C R}=\left(\left(l_{110}-l_{\mathrm{am}}\right) / l_{110}\right) \times 100
$$

10 The crystallinity index provides an idea on the crystalline fraction in chitin and its 11 derivatives. The chitin exposed to adjunct treatment may be susceptible to 12 depolymerization with crystalline fractions reduced. The crystallinity of steam 13 explosion and depressurization treated chitin were reduced from 88 to $73 \%$. These 14 treated chitin samples were hydrolyzed with chitinases, and produced higher amount 15 of oligomers compared to untreated chitin. ${ }^{38}$ The chitin that has been treated with a combination of supercritical water and mechano-chemical grinding exhibited higher

17 reduction of $I_{C R}$ from 91 to $26 \% .^{35}$ llankovan et al. ${ }^{12}$ reported that chitin chemically 18 treated with phosphoric acid, sodium hydroxide and methanol showed more 19 amorphous nature with lower intensities of the 110 reflection. The adjunct treatment 20 seems to result in chitin with lower crystallinity, which is more amenable to enzyme 21 action in order to produce oligomers.

\section{Molecular weight}


1 Molecular weight $(\mathrm{M})$ of chitin oligomers can be expressed in various ways, such as

2 weight-average (Mw), number-average $(\mathrm{Mn})$, and viscosity-average (Mv) molecular

3 weights. $\mathrm{Mn}$ and $\mathrm{Mw}$ represent the total weight of the oligomers molecules, i.e

4 GlcNAc and GlcN, divided by the total number of its constituting molecules and a 5 sum of the weight fraction of each type of molecules multiplied by its molecular 6 weight, respectively. ${ }^{35} \mathrm{Mv}$ can be determined from Mark-Houwink equation:

$$
[\eta]=k(M v)^{\alpha}
$$

8 where [n] $\left(\mathrm{cm}^{3} \mathrm{~g}^{-1}\right)$ is the intrinsic viscosity of chitin measured by viscometer, $\mathrm{k}$ is $90.24 \mathrm{~cm}^{3} \mathrm{~g}^{-1}$ and $\alpha$ is 0.69 , where, both $\mathrm{k}$ and $\alpha$ are constant parameters of 10 chitin. $^{27,86}$

11 Measurement of the molecular weight of chitin and its oligomers commonly can be 12 done by gel permeation chromatography (GPC) or size exclusion chromatography 13 (SEC), which is generally equipped with refractive index detector, a GPC (gel 14 permeation chromatography) column, and a guard column. ${ }^{35,87-89}$ The relative mean molecular weight of the chitin is estimated by the Pullulan standard curve. ${ }^{35}$ Prior to measurement, samples for GPC require microfiltration (with $0.45 \mu \mathrm{m}$ filters).$^{13,90}$

17 The molecular weight is a significant parameter for effective hydrolysis of chitin. 18 Kurita et al. ${ }^{81}$ reported that the chitin molecular weight of $300 \mathrm{kDa}$ was effective for 19 enzymatic hydrolysis. It has already been mentioned that the adjunct treatments 20 applied may reduce the molecular weight of chitin prior to hydrolysis. Dziril et al. ${ }^{37}$ reported that the molecular weight of gamma-radiated chitin decreased to $60 \%$ when irradiated with $50 \mathrm{kGy}$, and it further decreased to $90 \%$ when the applied dose was 23210 kGy. The application of supercritical water treatment combined with mechano- 
1 chemical grinding reduced the molecular weight of chitin from $800 \mathrm{kDa}$ to $10 \mathrm{kDa}{ }^{35}$

2 Ramírez-Coutiño et al. ${ }^{39}$ obtained the molecular weight of $343.5 \mathrm{kDa}$ for $\alpha$-chitin with

$390 \%$ of solubility after treating it with alkali. This molecular weight decrease is mainly

4 due to the glycoside bond breaking caused by the treatment of chitin. ${ }^{37}$

5 On the other hand, the molecular weight of chitin oligomers produced by hydrolysis 6 is lower than native chitin. Kazami et $a l{ }^{45}$ reported that the number-average 7 molecular weight of both $\alpha$ - and $\beta$-chitin rapidly decreased to $13 \mathrm{kDa}$ and $10 \mathrm{kDa}$ 8 from $241 \mathrm{kDa}$ and $90 \mathrm{kDa}$, respectively, after $15 \mathrm{~min}$ of hydrolysis in $\mathrm{HCl}$. At the 9 same time, the weight-average molecular weight of $\alpha$ - and $\beta$-chitin also reduced to 10 approximately $16 \mathrm{kDa}$ from $330 \mathrm{kDa}$ and $250 \mathrm{kDa}$, respectively. Previous study 11 reported that the chitin oligomers with molecular weight of 1 to $3 \mathrm{kDa}$ was more 12 effective as antioxidant agents those with molecular weight of $1 \mathrm{kDa}$ and lower. ${ }^{32}$ 13 Thus, the biological effect of chitin oligomers is significantly dependent on the 14 molecular weight. ${ }^{91}$

\section{Oligomers yield}

16 Yield of the oligomers after hydrolysis is a significant parameter because it 17 determines the economic viability of the process. The yield can be expressed simply 18 as the percentage of the chitin hydrolyzed as follows:

$$
\% \text { Yield }=\frac{W_{1}-W_{2}}{W_{1}} \times 100
$$

20 where, $W_{1}$ and $W_{2}$ are the initial weight and weight of chitin after hydrolysis, 21 respectively. ${ }^{31,40}$ The yields reported earlier for acidic hydrolysis of chitin lie between 2210 and $21 \% .40,45$ These values are comparable with enzymatic hydrolysis. Ilankovan 
1 et al. $^{12}$ and Hongkulsup ${ }^{31}$ reported the yield of chitin oligomers hydrolyzed by

2 commercial enzymes were in a range of $10 \%$ to $13 \%$. Although this is a low yield, it

3 can be increased by modifying chitin structure prior to hydrolysis by employing some

4 of the adjunct methods discussed in this paper.

5 High performance liquid chromatography (HPLC) is an efficient technique for 6 quantification of the oligomers according to each DP.6,11,31 The amount of each DP 7 (GICNACn) can be estimated with the calibration curve, as shown in the following 8 equation:

$$
C_{1}=C_{2} \times\left(A_{1} / A_{2}\right)
$$

10 where, $C_{1}$ is the sample concentration $(\mathrm{mg} / \mathrm{mL}), \mathrm{C}_{2}$ is the standard concentration $11(\mathrm{mg} / \mathrm{mL}), A_{1}$ is the peak area of sample and $A_{2}$ is the peak area of standard. ${ }^{6}$ Wang 12 et al. ${ }^{6}$ found the yield of the GlcNAc2, GlcNAc 3 , GlcNAc4 and GlcNAc5 were 0.44 $13 \mathrm{mg} / \mathrm{mL}, 0.08 \mathrm{mg} / \mathrm{mL}, 0.09 \mathrm{mg} / \mathrm{mL}$ and $0.43 \mathrm{mg} / \mathrm{mL}$, respectively, after chitin depolymerizing under fermentation condition for two days. The results obtained show that the concentrations of $\mathrm{GlcNAc}_{2}$ and $\mathrm{GlcNAc}_{5}$ are significantly higher than $\mathrm{GlcNAc}_{3}$ and GlcNAc 4 . Unlike fermentation, the hydrolysis of chitin by commercial 17 enzymes like hemicellulase and pectinase produce higher amounts of GlcNAc3, 18 GlcNAc$_{4}$ and GlcNAc5 $_{5}$ as reported by Hongkulsup ${ }^{31}$. In acid hydrolysis, higher acid 19 concentration and temperature can influence the amount of chitin oligomers formed. 20 The amount of $\mathrm{GlcNAc}_{2}, \mathrm{GlcNAc}_{3}$ and GlcNAc5 apparently increased when the acid 21 concentration increased from $4 \mathrm{~N}$ to $7 \mathrm{~N}$ during hydrolysis at $70{ }^{\circ} \mathrm{C} .40$ The amount of 22 GlcNAc2 produced can be 30 times greater when the hydrolysis temperature is 23 raised to $90{ }^{\circ} \mathrm{C} .40$ 


\section{CONCLUSION}

2 Chitin subjected to adjunct treatments allows modification of the native structure, and

3 improve the hydrolysis rate and product functionality. Microwave irradiation on the

4 chitin enhances access to the susceptible bonds for enzymatic hydrolysis and

5 reduces the reaction time for hydrolysis. Crystallinity and DA of the chitin reduced

6 when steam explosion was applied prior enzymatic hydrolysis. Furthermore, gamma

7 irradiation, and the combination of supercritical water and grinding, can reduce chitin

8 molecular weight and partially depolymerize it in order to facilitate enzyme attack on

9 the substrate. It is somewhat unfortunate that the literature reviewed in this study

10 does not specifically state the extent of yield improvement with statistical confidence,

11 which would have enabled us to establish commercial and economic viability of

12 using adjunct treatments. Nevertheless, one can conclude that chitin polymer

13 reacting with acid or enzymes, particularly $\mathrm{HCl}$ or chitinases, can successfully

14 produce oligomers with DP between 2 and 6 . The chitin oligomers are also reported

15 to have a potential to be commercialized for further applications, specifically as

16 antimicrobial agents. This is due to the simple preparation method and the quality of

17 oligomers produced without the need for deacetylation into chitosan. Unlike acid, the enzymatic hydrolysis may be considered to be more favourable due to the use of milder reaction conditions and environmental compatibility. Commercial enzymes may also be used to simplify the process and improve economics by reducing the

21 cost of specific enzymes extraction and purification.

22 In conclusion, all adjunct treatments reported so far, enhance the hydrolysis of chitin,

23 regardless of the hydrolysis method employed. For future research, it would be worth 24 trying pre-treatments like the application of high-pressure to chitin in order to lower 
1 its crystallinity. At the same time, it is also important to statistically quantify the

2 improvements produced by such treatments so that their viability can be conclusively 3 established.

4 


\section{References}

1 Feng $\mathrm{F}$, Liu $\mathrm{Y}$ and $\mathrm{Hu} \mathrm{K}$, Influence of alkali-freezing treatment on the solid state structure of chitin. Carbohydr Res 339: 2321-2324 (2004).

2 Jang MK, Kong BG, Jeong $\mathrm{YI}$, Lee $\mathrm{CH}$ and Nah JW, Physicochemical characterization of $\alpha$-chitin, $\beta$-chitin, and $\gamma$-chitin separated from natural resources. $J$ Polym Sci A Polym Chem 42: 3423-3432 (2004).

3 Arbia W, Arbia L, Adour L, and Amrane A, Chitin extraction from crustacean shells using biological methods - a review. Food Technol Biotechnol, 51:12-25 (2013).

4 Jeon YJ, Shahidi F and Kim SK, Preparation of chitin and chitosan oligomers and their applications in physiological functional foods. Food Rev Int 16: 159-176 (2000).

5 Gopalan NK and Dufresne A, Crab shells chitin whiskers reinforced natural rubber nanocomposites. 1. Processing and swelling behavior. Biomacromolecules 4: 657-665 (2003).

6 Wang SL, Liu CP and Liang TW, Fermented and enzymatic production of chitin/chitosan oligosaccharides by extracellular chitinases from Bacillus cereus TKU027. Carbohydr Polym 90: 1305-1313 (2012).

7 Kurita K, Chitin and chitosan: functional biopolymers from marine crustaceans. Mar Biotechnol 8: 203-226 (2006). 
8 Benhabiles MS, Abdi N, Drouiche N, Lounici H, Pauss A, Goosen MFA and Mameri N, Protein recovery by ultrafiltration during isolation of chitin from shrimp shells Parapenaeus longirostris. Food Hydrocoll 32: 28-34 (2013).

9 Synowiecki J and Al-Khateeb NA, Production, properties, and some new applications of chitin and its derivatives. Crit Rev Food Sci Nutr 43: 145-171 (2003).

10 Hirano S and Nagao N, An improved method for the preparation of colloidal chitin by using methanesulfonic acid. Agric Biol Chem 52: 2111-2112 (1988).

11 Il'ina AV, Zueva OY, Lopatin SA and Varlamov VP, Enzymatic hydrolysis of $\alpha$ chitin. Appl Biochem Microbiol 40: 35-38 (2004).

12 llankovan $\mathrm{P}$, Hein $\mathrm{S}, \mathrm{Ng} \mathrm{CH}$, Trung TS and Stevens WF, Production of $\mathrm{N}$ acetyl chitobiose from various chitin substrates using commercial enzymes. Carbohydr Polym 63: 245-250 (2006).

13 Kubota N, Tatsumoto N, Sano T and Toya K, A simple preparation of half Nacetylated chitosan highly soluble in water and aqueous organic solvents. Carbohydr Res 324: 268-274 (2000).

14 Rinaudo M, Chitin and chitosan: properties and applications. Progr Polym Sci 31: 603-632 (2006).

15 Kumar MNR, A review of chitin and chitosan applications. React Funct Polym 46: 1-27 (2000). 
16 Suh JKF and Matthew HW, Application of chitosan-based polysaccharide biomaterials in cartilage tissue engineering: a review. Biomater 21: 2589-2598 (2000).

17 Sogias IA, Khutoryanskiy VV and Williams AC, Exploring the factors affecting the solubility of chitosan in water. Macromol Chem Phys 211: 426-433 (2010).

18 Huei CR and Hwa HD, Effect of molecular weight of chitosan with the same degree of deacetylation on the thermal, mechanical, and permeability properties of the prepared membrane. Carbohydr Polym 29: 353-358 (1996).

19 Park SY, Marsh KS and Rhim JW, Characteristics of different molecular weight chitosan films affected by the type of organic solvents. J Food Sci 67: 194197 (2002).

20 Seyfarth F, Schliemann S, Elsner P and Hipler UC, Antifungal effect of highand low-molecular-weight chitosan hydrochloride, carboxymethyl chitosan, chitosan oligosaccharide and N-acetyl-D-glucosamine against Candida albicans, Candida krusei and Candida glabrata. Int J Pharm 353: 139-148 (2008).

21 Benhabiles MS, Drouiche N, Lounici H, Pauss A and Mameri N, Effect of shrimp chitosan coatings as affected by chitosan extraction processes on postharvest quality of strawberry. J Food Meas Charact 7: 215-221 (2013).

22 Vila A, Sánchez A, Janes K, Behrens I, Kissel T, Jato JLV and Alonso MJ, Low molecular weight chitosan nanoparticles as new carriers for nasal vaccine delivery in mice. Eur J Pharm Biopharm 57: 123-131 (2004). 
23 Xing R, Liu S, Guo Z, Yu H, Wang P, Li C, Li Z and Li P, Relevance of molecular weight of chitosan and its derivatives and their antioxidant activities in vitro. Bioorg Med Chem 13: 1573-1577 (2005).

24 Tsai GJ, Zhang SL and Shieh PL, (2004). Antimicrobial activity of a lowmolecular-weight chitosan obtained from cellulase digestion of chitosan. $J$ Food Prot 67: 396-398 (2004).

25 Prashanth $\mathrm{KVH}$ and Tharanathan RN, Chitin/chitosan: modifications and their unlimited application potential - an overview. Trends Food Sci Technol 18: 117-131 (2007).

26 Ahmed ABA, Taha RM, Mohajer S, Elaagib ME and Kim SK, Preparation, properties and biological applications of water soluble chitin oligosaccharides from marine organisms. Russ J Mar Biol 38: 351-358 (2012).

27 Benhabiles MS, Salah R, Lounici H, Drouiche N, Goosen MFA and Mameri N, Antibacterial activity of chitin, chitosan and its oligomers prepared from shrimp shell waste. Food Hydrocolloids 29: 48-56 (2012).

28 Alam $\mathrm{J}$ and Mathur A, Evaluation of antifungal potential of chitin and chitinbased derivatives against pathogenic fungal strains. Int $Q J$ Biol Life Sci 2: 13541358 (2014).

29 Liang TW, Chen YJ, Yen YH and Wang SL, The antitumor activity of the hydrolysates of chitinous materials hydrolyzed by crude enzyme from Bacillus amyloliquefaciens V656. Process Biochem 42: 527-534 (2007). 
30 Ngo DN, Kim MM and Kim SK, Chitin oligosaccharides inhibit oxidative stress in live cells. Carbohydr Polym 74: 228-234 (2008).

31 Hongkulsup C, Enzyme assisted extraction of chitin from shrimp shells (Litopenaeus vannamei) and its application as an antimicrobial agent. PhD thesis, University of Reading, UK, (2016).

32 Ngo DN, Lee SH, Kim MM and Kim SK, Production of chitin oligosaccharides with different molecular weights and their antioxidant effect in RAW 264.7 cells. J Funct Foods 1: 188-198 (2009).

33 Roy I, Mondal K and Gupta MN, Accelerating enzymatic hydrolysis of chitin by microwave pretreatment. Biotechnol Prog 19: 1648-1653 (2003).

34 Ajavakom A, Supsvetson S, Somboot A and Sukwattanasinitt, Products from microwave and ultrasonic wave assisted acid hydrolysis of chitin. Carbohydr Polym 90: 73-77 (2012).

35 Osada M, Miura C, Nakagawa YS, Kaihara M, Nikaido M and Totani K, Effects of supercritical water and mechanochemical grinding treatments on physicochemical properties of chitin. Carbohydr polym 92: 1573-1578 (2013).

36 Villa-Lerma G, González-Márquez H, Gimeno M, López-Luna A, Bárzana E and Shirai K, Ultrasonication and steam-explosion as chitin pretreatments for chitin oligosaccharide production by chitinases of Lecanicillium lecanii. Bioresour Technol 146: $794-798$ (2013). 
37 Dziril M, Grib H, Laribi-Habchi H, Drouiche N, Abdi N, Lounici H, Pauss A and Mameri $\mathrm{N}$, Chitin oligomers and monomers production by coupling $\mathrm{Y}$ radiation and enzymatic hydrolysis. J Ind Eng Chem 26: 396-401 (2015).

38 Villa-Lerma G, González-Márquez H, Gimeno M, Trombotto S, David L, Ifuku $\mathrm{S}$ and Shirai K, Enzymatic hydrolysis of chitin pretreated by rapid depressurization from supercritical 1, 1, 1, 2-tetrafluoroethane toward highly acetylated oligosaccharides. Bioresour Technol 209: 180-186 (2016).

39 Ramírez-Coutiño L, del Carmen Marín-Cervantes M, Huerta S, Revah S and Shirai K, Enzymatic hydrolysis of chitin in the production of oligosaccharides using Lecanicillium fungicola chitinases. Process Biochem 41: 1106-1110 (2006).

40 Chang KLB, Lee $\mathrm{J}$ and $\mathrm{Fu}$ WR, HPLC analysis of N-acetyl-chitooligosaccharides during the acid hydrolysis of chitin. J Food Drug Anal 8: 75-83 (2000).

41 Einbu $\mathrm{A}$ and Vårum $\mathrm{KM}$, Depolymerization and de- $\mathrm{N}$-acetylation of chitin oligomers in hydrochloric acid. Biomacromolecules 8: 309-314 (2007).

42 Trombotto $\mathrm{S}$, Ladavière $\mathrm{C}$, Delolme $\mathrm{F}$ and Domard $\mathrm{A}$, Chemical preparation and structural characterization of a homogeneous series of chitin/chitosan oligomers. Biomacromolecules 9: 1731-1738 (2008).

43 Einbu $A$ and Vårum KM, Characterization of chitin and its hydrolysis to GlcNAc and GlcN. Biomacromolecules 9: 1870-1875 (2008).

44 Einbu A, Grasdalen H and Vårum KM, Kinetics of hydrolysis of chitin/chitosan oligomers in concentrated hydrochloric acid. Carbohydr Res 342: 1055-1062 (2007). 
45 Kazami N, Sakaguchi M, Mizutani D, Masuda T, Wakita S, Oyama F, Kawakita $\mathrm{M}$ and Sugahara $\mathrm{Y}, \mathrm{A}$ simple procedure for preparing chitin oligomers through acetone precipitation after hydrolysis in concentrated hydrochloric acid. Carbohydr Polym 132: 304-310 (2015).

46 Moerschbacher BM and El Gueddari NE, Bio-activity matrices for partially acetylated chitosan oligomers, Proc. ICCC-EUCHIS2006 Montpellier, France, ed. Domard A, Guibal E and Vårum KM, Advances in Chitin Science (as CD Rom), pp 10-23 (2007).

47 Purushotham P, Sarma PVSRN and Podile AR, Multiple chitinases of an endophytic Serratia proteamaculans 568 generate chitin oligomers. Bioresour Technol 112: 261-269 (2012).

48 Eva-Lena HULT, Katouno F, Uchiyama T, Watanabe T and Sugiyama J, Molecular directionality in crystalline $\beta$-chitin: hydrolysis by chitinases $A$ and $B$ from Serratia marcescens 2170. Biochem J 388: 851-856 (2005).

49 Hamer SN, Cord-Landwehr S, Biarnés $\mathrm{X}$, Planas $\mathrm{A}$, Waegeman $\mathrm{H}$, Moerschbacher BM and Kolkenbrock S, Enzymatic production of defined chitosan oligomers with a specific pattern of acetylation using a combination of chitin oligosaccharide deacetylases. Sci Rep 5: 8716.

50 Terwisscha van Scheltinga AC, Armand S, Kalk KH, Isogai A, Henrissat B and Dijkstra BW, Stereochemistry of chitin hydrolysis by a plant chitinase/lysozyme and x-ray structure of a complex with allosamidin evidence for substrate assisted catalysis. Biochem 34: 15619-15623 (1995). 
51 Roy I, Sardar M and Gupta MN, Hydrolysis of chitin by Pectinex ${ }^{\mathrm{TM}}$. Enzyme Microb Technol 32: 582-588 (2003).

52 Das S, Sen R and Roy D, Enzymatic processing of chitinaceous wastes for Nacetyl-d-glucosamine production: an example of green and efficient environmental management. Environ Eng Manag J 11: 1849-1855 (2012).

53 Machová E, Kvapilova K, Kogan G and Šandula J, Effect of ultrasonic treatment on the molecular weight of carboxymethylated chitin-glucan complex from Aspergillus niger. Ultrason Sonochem 5: 169-172 (1999).

54 Takahashi Y, Miki F and Nagase K, Effect of sonolysis on acid degradation of chitin to form oligosaccharides. Bull Chem Soc Jpn 68: 1851-1857 (1995).

55 Kjartansson GT, Zivanovic S, Kristbergsson $\mathrm{K}$ and Weiss J, Sonicationassisted extraction of chitin from shells of fresh water prawns (Macrobrachium rosenbergii)). J Agric Food Chem 54: 3317-3323 (2006).

56 Wu HS, Lin BC and Sitanggang AB, Process for producing glucosamine and acetyl glucosamine by microwave technique. US Patent 20110114472 A1 (20011).

57 Choi WS, Ahn KJ, Lee DW, Byun MW and Park HJ, Preparation of chitosan oligomers by irradiation. Polym Degrad Stab 78: 533-538 (2002).

58 Hai L, Diep TB, Nagasawa N, Yoshii $\mathrm{F}$ and Kume T, Radiation depolymerization of chitosan to prepare oligomers. Nucl Instrum Methods Phys Res Sect B 208: 466-470 (2003). 
59 Kang B, Dai YD, Zhang $H Q$ and Chen D, Synergetic degradation of chitosan with gamma radiation and hydrogen peroxide. Polym Degrad Stab 92: 359-362 (2007).

60 Oliveira FM, Pinheiro IO, Souto-Maior AM, Martin C, Gonçalves AR and Rocha GJ, Industrial-scale steam explosion pretreatment of sugarcane straw for enzymatic hydrolysis of cellulose for production of second generation ethanol and value-added products. Bioresour Technol 130; 168-173 (2013).

61 Alvira P, Negro MJ, Ballesteros I, González A and Ballesteros M, Steam explosion for wheat straw pretreatment for sugars production. Bioethanol 2: 66-75 (2016).

62 Savage PE, Organic chemical reactions in supercritical water. Chem Rev 99: 603-622 (1999).

63 Zaluski L, Zaluska A and Ström-Olsen JO, Hydrogenation properties of complex alkali metal hydrides fabricated by mechano-chemical synthesis. J Alloys Compd 290: 71-78 (1999).

64 Osada M, Miura C, Nakagawa YS, Kaihara M, Nikaido M and Totani K, Effect of sub-and supercritical water pretreatment on enzymatic degradation of chitin. Carbohydr Polym 88: 308-312 (2012).

65 Cho YW, Jang J, Park C R and Ko SW, Preparation and solubility in acid and water of partially deacetylated chitins. Biomacromolecules 1: 609-614 (2000).

66 Kurita K, Controlled functionalization of the polysaccharide chitin. Prog Polym Sci 26: 1921-1971 (2001). 
67 Kasaai MR, A review of several reported procedures to determine the degree of $\mathrm{N}$-acetylation for chitin and chitosan using infrared spectroscopy. Carbohydr Polym 71: 497-508 (2008).

68 Duarte ML, Ferreira MC, Marvao MR and Rocha J, An optimised method to determine the degree of acetylation of chitin and chitosan by FTIR spectroscopy. Int J Biol Macromo/ 31: 1-8 (2002).

69 Stuart BH, Experimental methods, in Infrared Spectroscopy: Fundamentals and Applications, ed by Stuart BH, John Wiley \& Sons Ltd, Chichester, UK, pp 76-77 (2004).

70 Van de Velde $\mathrm{K}$ and Kiekens $\mathrm{P}$, Structure analysis and degree of substitution of chitin, chitosan and dibutyrylchitin by FT-IR spectroscopy and solid state $13 \mathrm{C}$ NMR. Carbohydr Polym 58: 409-416 (2004).

71 Brugnerotto J, Lizardi J, Goycoolea FM, Argüelles-Monal W, Desbrieres J and Rinaudo M, An infrared investigation in relation with chitin and chitosan characterization. Polym 42: 3569-3580 (2001).

72 Knight JA, Smoak MP, Porter RA and Kirkland WE, Direct pressing of fibers for infrared spectroscopic studies. Text Res J 37: 924-927 (1967).

73 Kaya M, Sargin I, Tozak KÖ, Baran T, Erdogan S and Sezen G, Chitin extraction and characterization from Daphnia magna resting eggs. Int $\mathrm{J}$ Biol Macromol 61: 459-464 (2013). 
74 Al Sagheer FA, AI-Sughayer MA, Muslim S and Elsabee MZ, Extraction and characterization of chitin and chitosan from marine sources in Arabian Gulf. Carbohydr Polym 77: 410-419 (2009).

75 Kaya M, Baran T, Mentes A, Asaroglu M, Sezen G and Tozak KO, Extraction and characterization of $\alpha$-chitin and chitosan from six different aquatic invertebrates. Food Biophys 9: 145-157 (2014).

76 Beil S, Schamberger A, Naumann W, Machill $S$ and van Pée $\mathrm{KH}$, Determination of the degree of $\mathrm{N}$-acetylation (DA) of chitin and chitosan in the presence of water by first derivative ATR FTIR spectroscopy. Carbohydr Polym 87: 117-122 (2012).

77 Wu T and Zivanovic S, Determination of the degree of acetylation (DA) of chitin and chitosan by an improved first derivative UV method. Carbohydr Polym 73: 248-253 (2008).

78 Baxter A, Dillon M, Taylor KA and Roberts GA, Improved method for IR determination of the degree of N-acetylation of chitosan. Int J Biol Macromol 14: 166169 (1992).

79 Ottey $\mathrm{MH}$, Vårum $\mathrm{KM}$ and Smidsrød $\mathrm{O}$, Compositional heterogeneity of heterogeneously deacetylated chitosans. Carbohydr Polym 29: 17-24 (1996).

80 Hongkulsup C, Khutoryanskiy VV and Niranjan K, Enzyme assisted extraction of chitin from shrimp shells (Litopenaeus vannamei). J Chem Technol Biotechnol 91: 1250-1256 (2016). 
81 Kurita K, Kaji Y, Mori T and Nishiyama Y, Enzymatic degradation of $\beta$-chitin: susceptibility and the influence of deacetylation. Carbohydr Polym 42: 19-21 (2000).

82 Cabrera JC and Van Cutsem P, Preparation of chitooligosaccharides with degree of polymerization higher than 6 by acid or enzymatic degradation of chitosan. Biochem Eng J 25: 165-172 (2005).

83 Li J, Du Y, Yang J, Feng T, Li A and Chen P, Preparation and characterisation of low molecular weight chitosan and chito-oligomers by a commercial enzyme. Polym Degrad Stab 87: 441-448 (2005).

84 Nars A, Rey T, Lafitte C, Vergnes S, Amatya S, Jacquet C, Dumas B, Thibaudeau C, Heux L, Bottin A and Fliegmann J, An experimental system to study responses of Medicago truncatula roots to chitin oligomers of high degree of polymerization and other microbial elicitors. Plant cell Rep 32: 489-502 (2013).

85 Salaberria AM, Fernandes SC, Diaz RH and Labidi J, Processing of $\alpha$-chitin nanofibers by dynamic high pressure homogenization: characterization and antifungal activity against $A$. niger. Carbohydr polym 116: 286-291 (2015).

86 Mirzadeh H, Yaghobi N, Amanpour S, Ahmadi H, Mohagheghi M A and Hormozi F, Preparation of chitosan derived from shrimp's shell of Persian Gulf as a blood hemostasis agent. Iran Polym J 11: 63-68 (2002).

87 Teng WL, Khor E, Tan TK, Lim LY and Tan SC, Concurrent production of chitin from shrimp shells and fungi. Carbohydr Res 332: 305-316 (2001).

88 Qin $C Q$, Du YM and Xiao L, Effect of hydrogen peroxide treatment on the molecular weight and structure of chitosan. Polym Degrad Stab 76: 211-218 (2002). 
89 Yen MT, Yang JH and Mau JL, Physicochemical characterization of chitin and chitosan from crab shells. Carbohydr Polym 75: 15-21 (2009).

90 Kasaai MR, Arul $J$ and Charlet G, Intrinsic viscosity-molecular weight relationship for chitosan. J Polym Sci Part B: Polym Phys 38: 2591-2598 (2000).

91 No HK, Park NY, Lee SH and Meyers SP, Antibacterial activity of chitosans and chitosan oligomers with different molecular weights. Int J Food Microbiol 74: 65$72(2002)$.

92 Salah R, Michaud P, Mati F, Harrat Z, Lounici H, Abdi N, Drouiche N, Mameri $\mathrm{N}$, Anticancer activity of chemically prepared shrimp low molecular weight chitin evaluation with the human monocyte leukaemia cell line, THP-1. Int J Biol Macromol 52:333-339 (2013). 